OPEN ACCESS

Edited by:

Yuchen Liu,

Shenzhen University, China

Reviewed by:

Tingting Dai,

Nanjing Forestry University, China

Syed Khizer Hasan,

Advanced Centre for Treatment, Research and Education in Cancer,

India

*Correspondence:

Xinjie Wang

wang_xin_jie@126.com

Fuling Zhou

zhoufuling@whu.edu.cn

Specialty section:

This article was submitted to

Molecular Diagnostics and

Therapeutics,

a section of the journal

Frontiers in Molecular Biosciences

Received: 28 August 2021

Accepted: 11 October 2021

Published: 25 October 2021

Citation:

Liu Y, Chen Y, Huang S, Ma X, Huang $X$, Wang $X$ and Zhou $F$ (2021) Rapid and Sensitive Diagnosis of DrugResistant FLT3-F691L Mutation by

CRISPR Detection

Front. Mol. Biosci. 8:753276.

doi: $10.3389 /$ fmolb.2021.753276

\section{Rapid and Sensitive Diagnosis of Drug-Resistant FLT3-F691L Mutation by CRISPR Detection}

\author{
Yin Liu ${ }^{1}$, Yanling Chen ${ }^{1}$, Shisheng Huang ${ }^{2}$, Xiaodong $\mathrm{Ma}^{3}$, Xingxu Huang ${ }^{2}$, Xinjie Wang ${ }^{3,4 *}$ \\ and Fuling Zhou ${ }^{1 *}$
}

${ }^{1}$ Department of Hematology, Zhongnan Hospital of Wuhan University, Wuhan, China, ${ }^{2}$ School of Life Science and Technology, ShanghaiTech University, Shanghai, China, ${ }^{3}$ Key Laboratory of Brain, Cognition and Education Sciences, Institute for Brain Research and Rehabilitation, South China Normal University, Ministry of Education, Guangzhou, China, ${ }^{4}$ Shenzhen Branch, Guangdong Laboratory of Lingnan Modern Agriculture, Genome Analysis Laboratory of the Ministry of Agriculture and Rural Affairs, Agricultural Genomics Institute at Shenzhen, Chinese Academy of Agricultural Sciencesn, Shenzhen, China

Sensitive and efficient detection of drug-resistant mutations is essential in cancer precision medicine. In treating acute myeloid leukemia (AML), FLT3 gene F691L mutation shows universal resistance to all currently available FLT3 inhibitors. However, there is no particular detection method for FLT3-F691L. Commonly-used first-generation sequencing (FGS) approaches have low sensitivity, and next-generation sequencing (NGS) is timeconsuming. Herein, we developed an accurate and sensitive FLT3-F691L diagnostic method by CRISPR detection. Briefly, the FLT3-691 region is amplified by recombinase polymerase amplification (RPA) and detected by L691-crRNA induced Cas12a reaction, and finally the result can be directly observed under a blue lamp or analyzed by a fluorescence reader. Confirmed by the tests on diluted plasmids and 120 AML patient samples, this method can achieve a sensitivity of $0.1 \%$ and complete the whole diagnosis process within $40 \mathrm{~min}$. Potentially, this method will play an important role in point-of-care applications and guidance of AML treatment.

Keywords: drug resistance, acute myeloid leukemia, FMS-like tyrosine kinase 3, F691L mutation, CRISPR detection

\section{INTRODUCTION}

Acute myeloid leukemia (AML), similar to many other cancers, is characterized by various somatically acquired mutations that affect gene functions (Dohner et al., 2021). FMS-like tyrosine kinase 3 (FLT3) gene mutations were observed in approximately 30\% of AML cases, including 25\% internal tandem duplication (ITD) and 7-10\% tyrosine kinase domain (TKD) mutation (Daver et al., 2019). The deep understanding of FLT3 mutations leads to the emergence of multiple FLT3 inhibitors, which are now one of the most promising treatments for AML (Kiyoi et al., 2020). The first-generation FLT3 inhibitors are multi-targeted, include midostaurin, sunitinib, lestaurtinib, sorafenib, and tandutinib. The second-generation FLT3 inhibitors are more selective and effective as single agents, represented by gilteritinib, crenolanib, and quizartinib. Most FLT3 mutated cases are sensitive to at least one of them. However, a "gatekeeper" mutation FLT3-F691L shows universal resistance to all these inhibitors (Eguchi et al., 2020; Smith et al., 2015; Tarver et al., 2020). Therefore, the early detection of FLT3-F691L mutation is of vital importance in avoiding ineffective treatment for AML patients.

Currently-used FLT3-F691L detection methods include first-generation sequencing (FGS) and next-generation sequencing (NGS) approaches, and both have strengths and limitations. FGS is now 
a well-established technology that can output results within 1 day, but its low sensitivity limits the detection of a mutation rate of less than $10 \%$. On the other hand, NGS is much more sensitive and accurate. However, NGS is too time-consuming (more than 1 week) and not economical. Therefore, a rapid and sensitive method for FLT3-F691L detection is still needed.

Recently, the clustered regularly interspaced short palindromic repeats (CRISPR) based nucleic acid detection was developed, with the advantages of sensitivity, specificity, and rapidity (Li et al., 2019). The core mechanism of CRISPR detection is that CRISPR-associated (Cas) proteins can specifically recognize targeted nucleic acids under the guidance of a single-stranded guide CRISPR RNA (crRNA). The most frequently used Cas proteins are Cas9, Cas12, and Cas13 (Gootenberg et al., 2017; Li et al., 2018a; Li et al., 2018b; Chen et al., 2018; Myhrvold et al., 2018; Zhou et al., 2018; Chen Q. et al., 2020; Wang et al., 2020b). Especially, Cas12a can generate a nonspecific ssDNAase activity upon target recognition, which allows Cas12a to be coupled with an ssDNA fluorescence reporter and construct an ultrasensitive molecular detection method (Wang et al., 2020b). To date, Cas12a-based detection technologies have been used to diagnose various infectious diseases, including SARS-CoV-2, African swine fever virus, Dengue virus, and Mycobacterium tuberculosis (Wang et al., 2020a; Curti et al., 2020; Wang et al., 2020c; Ding et al., 2020; Ma et al., 2020; Xu et al., 2020).

Herein, we combined Cas12a-based detection with recombinase polymerase amplification (RPA) to establish a sensitive, accurate and rapid system for FLT3-F691L detection. Potentially, this method will play an important role in point-ofcare applications and guidance of AML treatment.

\section{MATERIALS AND METHODS}

\section{Patient Samples}

Peripheral blood samples of 120 AML patients were collected from the Hematology Department in Zhongnan Hospital of Wuhan University under an approved Institutional Review Board protocol. For DNA template preparation, about $500 \mu \mathrm{l}$ of peripheral blood was mixed well with four times the volume of the RBC lysis buffer (Biosharp, Hefei, China). After 1 min of lysis, WBCs were precipitated by 1 min of mini centrifugation and then incubated with $150 \mu \mathrm{l}$ nucleic acid releaser (GenDx, Suzhou, China) at $95^{\circ} \mathrm{C}$ for $3 \mathrm{~min}$ to release genomic DNA. Two microliters of the released DNA were used for subsequent RPA.

\section{Plasmid and DNA Fragments Preparation}

A 630-bp DNA fragment covering the WT FLT3-691 site was amplified by PCR with primers $\mathrm{P} 1$ and $\mathrm{P} 2$ from a WT patient's genomic DNA, then cloned into the pGem-T vector (Takara, China). After transformation into Escherichia coli DH5a, the T-vec-F691 plasmid was extracted by an AxyPrep Plasmid Miniprep Kit (Axygen, CA, United States). For constructing the FLT3-L691 plasmid, primers P3 and P4 carrying a $\mathrm{T}>\mathrm{G}$ base mutation were used to amplify the T-vec-F691 plasmid circularly. Then the amplified product was self-ligated and cloned into the pGem-T vector to construct the T-vec-F691 plasmid. For identification of the specificity of crRNAs, 350-bp F691 and L691 DNA fragments were produced by PCR with primers P5 and P6 using T-vec-F691 and FLT3-L691 plasmids as templates. The quantification of the above plasmids and DNA fragments were conducted by a Nanodrop2000 (Thermo Fisher Scientific, MA, United States). The sequence information of PCR primers are listed in Supplementary Table S1.

\section{RPA}

The RPA assay was conducted by a GenDx ERA Kit (Suzhou GenDx Biotech, China). Briefly, the $50 \mu \mathrm{l}$ RPA system included $2 \mu \mathrm{l}$ DNA template, $2.5 \mu$ forward primer $(10 \mathrm{nM}), 2.5 \mu$ l reverse primer $(10 \mathrm{nM}), 11 \mu \mathrm{l}$ ERA basic buffer, $20 \mu \mathrm{l}$ reaction buffer, $2 \mu \mathrm{l}$ activator, and supplementary $\mathrm{dd} \mathrm{H} 2 \mathrm{O}$. After sufficient mixing, the mixture was incubated at $37^{\circ} \mathrm{C}$ for $15 \mathrm{~min}$. Five microliters of the RPA product were transferred to subsequent Cas12a reaction. The RPA primers are listed in Supplementary Table S1.

\section{Cas12a Fluorescence Reaction}

Cas12a fluorescence reaction was conducted according to a previous description (Wang et al., 2020a). The $20 \mu \mathrm{l}$ reaction mixture included $200 \mathrm{ng}$ Cas12a, $0.1 \mathrm{pmol}$ crRNA, $2 \mu \mathrm{l} 10 \times$ NEBuffer 3.1 (NEB, MA, United States), $1 \mu$ l RNase inhibitor (Novoprotein, China), 25 pmol ssDNA-FQ reporter (Genewiz, NJ, America), an appropriate amount of DNA fragments or $5 \mu \mathrm{l}$ RPA product, and supplementary ddH2O. Among these components, crRNAs were directly synthesized by GenScript (Nanjing, China). And the sequence information of F691crRNA and L691-crRNA are listed in Supplementary Table S2. Cas12a protein was produced as described previously (Creutzburg et al., 2020). The mixed Cas12a reaction system was incubated at $37^{\circ} \mathrm{C}$ for $15 \mathrm{~min}$, and then the result was directly observed under a 485-nm blue lamp (Sangon, Shanghai, China). Fluorescence kinetics were monitored using a monochromator with excitation at $485 \mathrm{~nm}$ and emission at $520 \mathrm{~nm}$.

\section{FGS and NGS}

For FGS detection, $30 \mu \mathrm{l}$ PCR products or $30 \mu \mathrm{l}$ RPA products were purified by the AxyPrep PCR Clean-up Kit and quantified by the Nanodrop2000. Approximately 200 ng DNA products were sent to FGS conducted by Tsingke (Beijing, China). Different barcoded primers were designed and synthesized for the PCR amplification of the FLT3-691 region of genomic DNA samples for NGS detection. The amplified products were then purified and mixed equally for NGS detection by the Illumina NextSeq $500(2 \times 150)$ platform at the CAS-MPG Partner Institute for Computational Biology Omics Core, Shanghai, China. The PCR primers for the preparation of NGS samples are listed in Supplementary Table S3.

\section{Statistical Analysis}

We have repeated all experiments three times. Statistical analysis was performed by GraphPad Prism software version 8.0. Unpaired two-tailed Student's t-test was used for comparison between two groups. Quantitative data are expressed as mean value \pm SE. ${ }^{*} p<0.05,{ }^{* *} p<0.01,{ }^{* * *} p<0.001,{ }^{* * *} p<0.0001$; ns, no significance. 

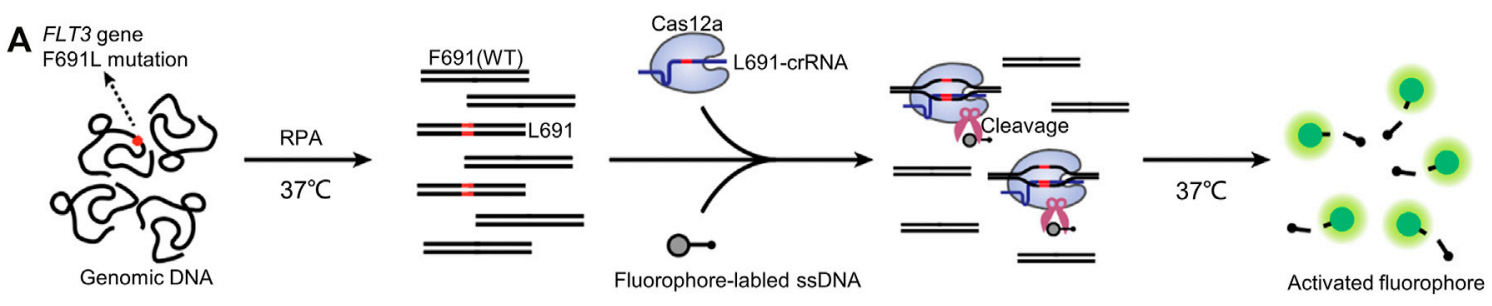

B

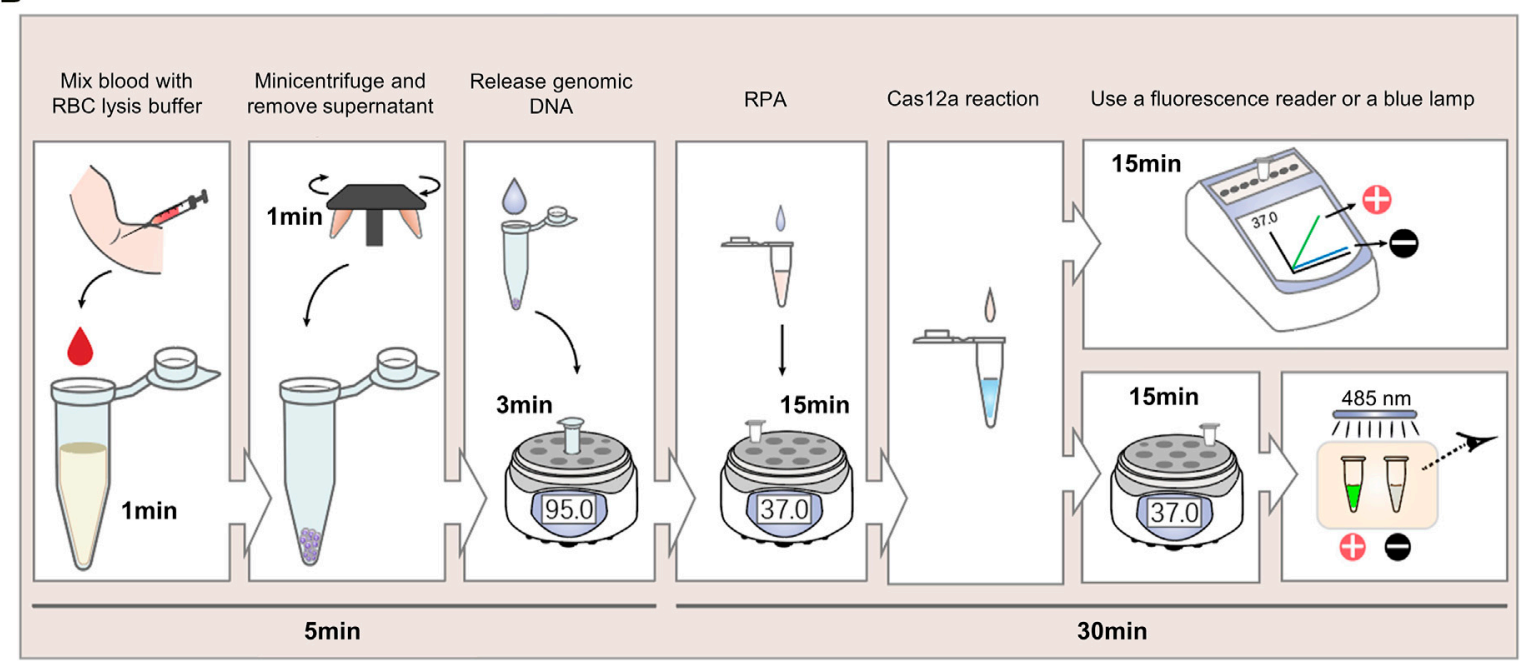

FIGURE 1 | The CRISPR detection system for FLT3-F691L diagnosis. (A) Schematic Diagram of the CRISPR detection method for FLT3-F691L diagnosis. (B) Flowcharts of the whole process from drawing blood to receiving the result in the detection of FLT3-F691L mutation by CRISPR detection.

\section{RESULTS}

\section{Development of the CRISPR Detection System for FLT3-F691L Diagnosis}

Commonly-used CRISPR detection includes two steps, isothermal amplification and Cas12a fluorescence reaction. Recombinase polymerase amplification (RPA) is a highly selective and efficient isothermal amplification technique, which allows the amplification of single-digit DNA target copies under $37-42^{\circ} \mathrm{C}$ (Lobato and O'Sullivan, 2018). In this study, RPA was adopted to amplify the FLT3-691 region of genomic DNA. Then RPA product was detected by L691-crRNA induced Cas12a reaction, wherein fluorophore-labeled ssDNA existed. Upon recognizing F691L mutant amplicons, Cas12a will generate nonspecific ssDNAase activity to cleave fluorophore-labeled ssDNA and release fluorescence signal (Figure 1A).

We developed a complete diagnosis process from drawing blood to receiving the clinical application of this CRISPR detection system. First, the blood drawn from the patient was mixed with red blood cell (RBC) lysis buffer for $1 \mathrm{~min}$. Then $1 \mathrm{~min}$ of centrifugation was adopted to obtain white blood cell (WBC) precipitation. Next, the genomic DNA of these WBCs was released by a nucleic acid releaser under $95^{\circ} \mathrm{C}$ for $3 \mathrm{~min}$. Then, the DNA sample was amplified by RPA under $37^{\circ} \mathrm{C}$ for $15 \mathrm{~min}$, and detected by L691-crRNA induced Cas12a reaction under $37^{\circ} \mathrm{C}$ for $15 \mathrm{~min}$. Finally, the result can be analyzed by a fluorescence reader or directly observed by naked eyes under a blue lamp. Increased fluorescence intensity and green fluorescence signal indicate a F691L positive result. On the contrary, unchanged fluorescence intensity and no green fluorescence signal suggest a F691L negative result (Figure 1B). Thus, the whole diagnosis can be completed within $40 \mathrm{~min}$, without the need for large equipment or expensive reagent.

\section{Identification of the Specificity of crRNA for F691L Detection}

The high specificity of the Cas12a reaction relies on a specific crRNA, which only recognizes target DNA while ignores nontargets. For the detection of mutation-type (MT) FLT3-L691 (c. TTG) from wild-type (WT) FLT3-F691 (c. TTT), we designed a crRNA with a sequence complementary to L691 genomic DNA (Figure 2A). To identify the specificity of L691-crRNA, we used L691-crRNA induced Cas12a reaction to detect 1e12 copies of WT and L691 DNA fragments, as well as other four mutations in the detection region, which were recorded in the catalogue of somatic mutations in cancer (COSMIC) database (Supplementary Table S4). After 15 min reaction under $37^{\circ} \mathrm{C}$, the L691 sample showed a strong fluorescence signal, and no fluorescence was observed in WT and other mutation samples. These results confirmed the high specificity of L691-crRNA (Figures 2B,C). Then gradient copies 


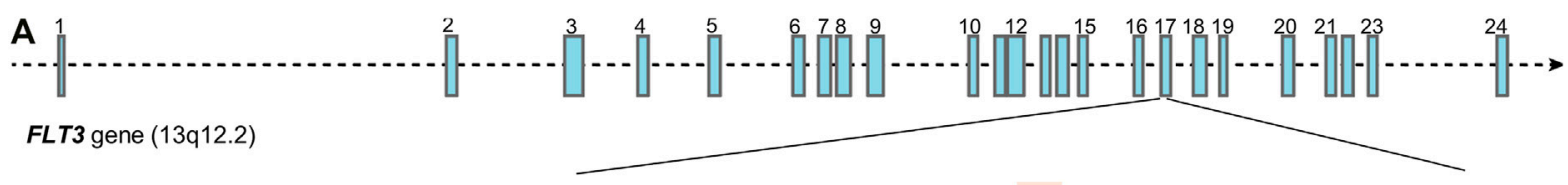

FLT3-F691(WT) genomic DNA 5’-TTTCAGGACCAATTTACTTGATTTTTGAATACTGTTGCTATGGTGATCTT-3’ FLT3-L691(MT) genomic DNA 5’-TTTCAGGACCAATTTACTTGATTTTGGAATACTGTTGCTATGGTGATCTT-3’

L691-crRNA - - - - CUUGAUUUUGGAAUACUGUUGCU

B

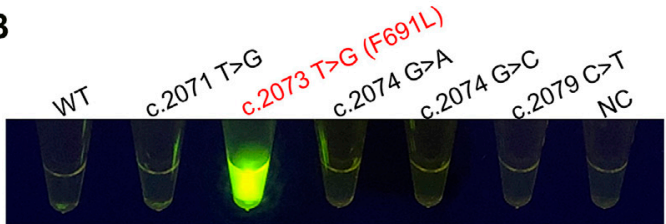

WT

c.2071 T>G 5' 5'-CAATTTACTTGATTGTTGAATACTGTTGCTATG-3' c.2073 T>G 5' -CAATTTACTTGATTTTGGAATACTGTTGCTATG-3' c.2074 G>A 5' -CAATTTACTTGATTTTTAAATACTGTTGCTATG-3' c.2074 G>C 5' -CAATTTACTTGATTTTTCAATACTGTTGCTATG-3' c.2079 C>T 5' 5'-CAATTTACTTGATTTTTGAATATTGTTGCTATG-3'

D

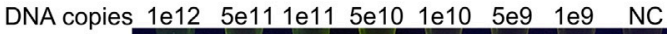
L691

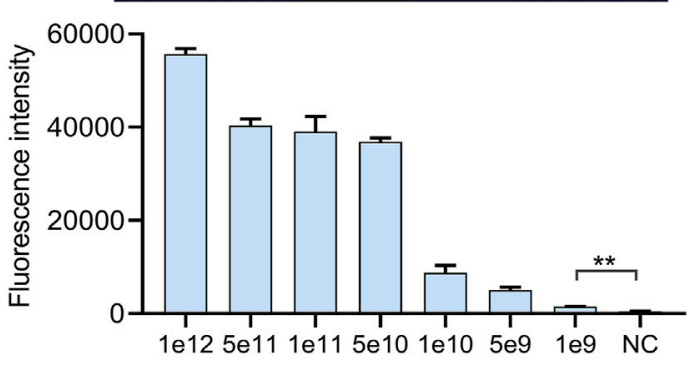

C

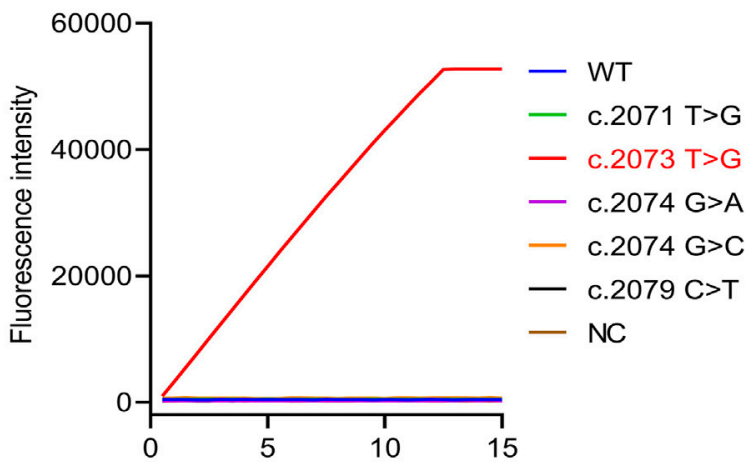

E

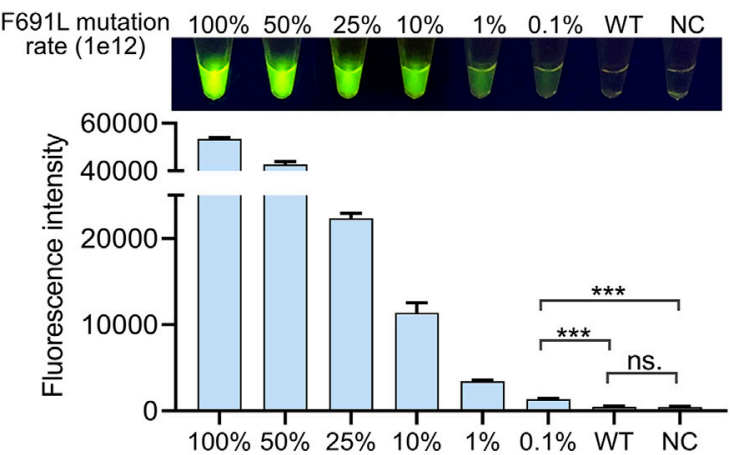

FIGURE 2 | Identification of the specificity of crRNA for F691 L detection. (A) FLT3 gene structure diagram, sequences of WT and mutant genomic DNA, and L691crRNA. Blue boxes show the 24 exons of FLT3 gene. FLT3-691 region in exon 17 is partially magnified. Green bases represent the specific protospacer adjacent motif (PAM) of Cas12a. The amino acid codon of 691 site is marked by orange boxes, and the T > G mutation is colored in red. (B) The naked-eye result of L691-crRNA induced Cas12a reaction in the detection of $1 \mathrm{e} 12$ copies of WT, F691L, and other four mutation DNA fragments. The reaction is under $37^{\circ} \mathrm{C}$ for $15 \mathrm{~min}$. NC, negative control. The sequences of these samples are shown below. (C) time-course analysis of (B). (D) The naked-eye result of L691-crRNA induced Cas12a reaction in the detection of gradient copies of L691 DNA fragments. The reaction is under $37^{\circ} \mathrm{C}$ for $15 \mathrm{~min}$. The statistical chart is shown below. (E) The naked-eye result of L691crRNA induced Cas12a reaction in the detection of $1 \mathrm{e} 12$ copies of DNA fragments with different F691L mutation rates. The reaction is under $37^{\circ} \mathrm{C}$ for 15 min. The statistical chart is shown below.

(1e12 1e9) of L691 DNA fragments were used to identify the sensitivity of L691-crRNA induced Cas12a reaction. The result showed that as low as $1 \mathrm{e} 9$ copies of L691 DNA fragments could be detected (Figure 2D). Finally, we mixed 1e12 copies of F691 and L691 DNA fragments with different proportions to simulate mutation rates $(100,50,25,10,1,0.1 \%$, and WT). As expected, the fluorescence signals gradually decreased with mutation rates. A significant difference could be observed between $0.1 \%$ sample and the negative control, while no significant difference was found between WT sample and the negative control. Together, these results indicated that L691-crRNA is highly specific for the detection of FLT3-F691L mutation (Figure 2E). In the same way, we also designed an F691-crRNA for the detection of WT FLT3-F691 (Supplementary Figrue S1).

\section{RPA Primer Screening for Highly Sensitive Detection}

To achieve an ultrasensitive detection of FLT3-F691L mutation, a highly efficient amplification is needed to produce enough target copies. According to the design principles of RPA primers, we designed four forward primers RPA-F1 $\sim 4$ and four reverse primers RPA-R1 $\sim 4$ for the amplification of the FLT3-691 region (Figure 3A). Then 16 primer pairs were generated by 

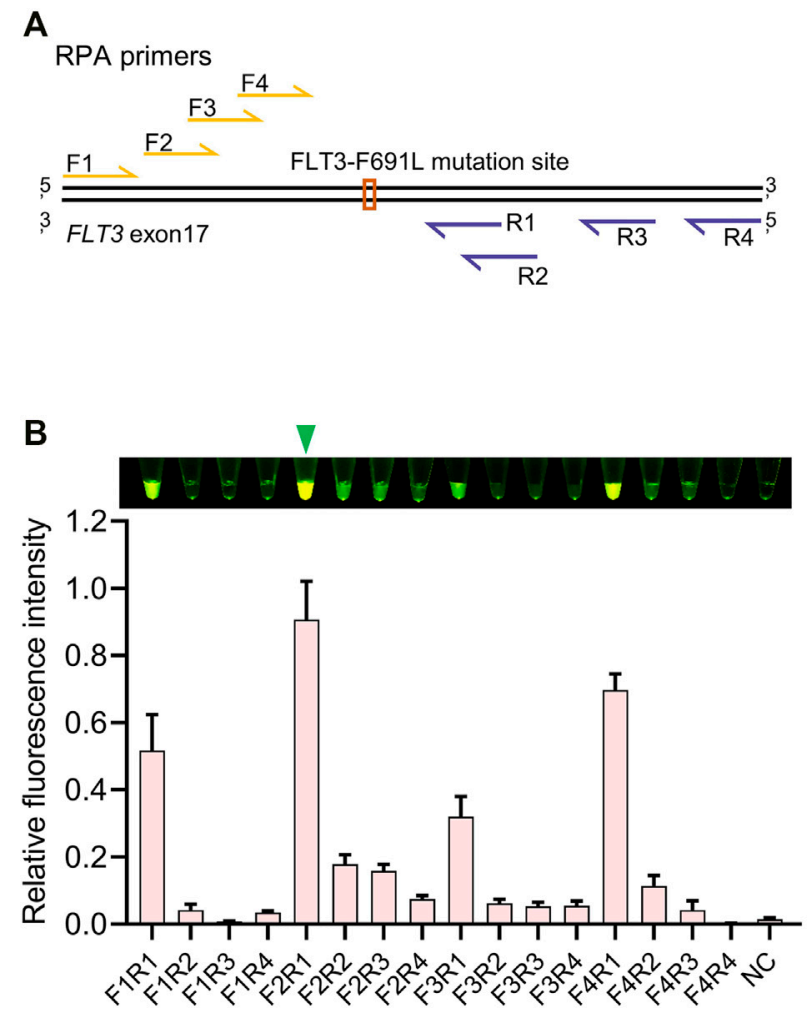

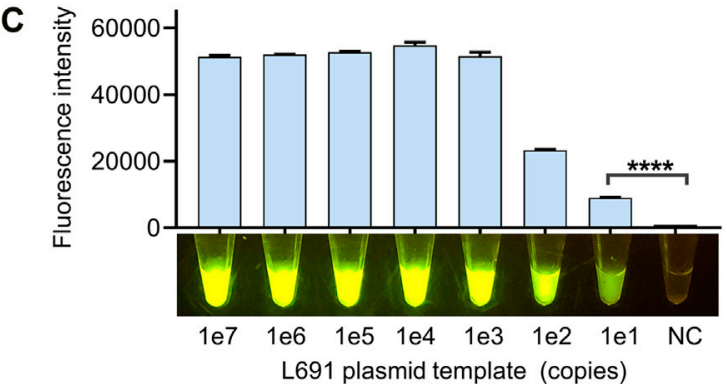

D

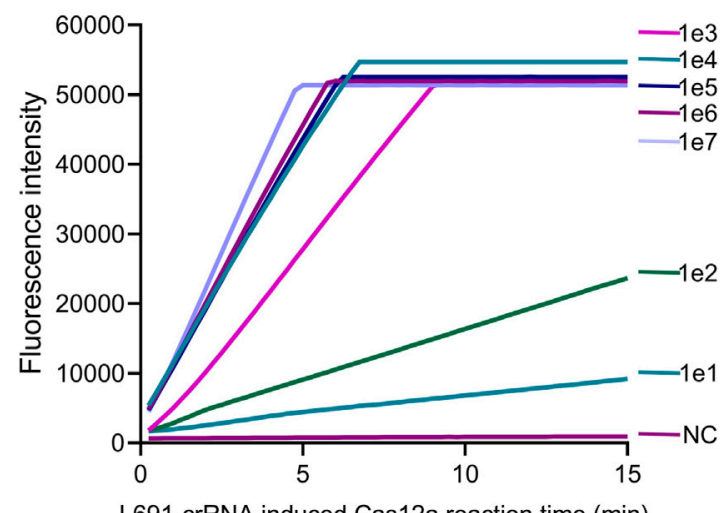

L691-crRNA induced Cas12a reaction time $(\mathrm{min})$

FIGURE 3 |RPA primer screen for highly sensitive detection. (A) Relative locations of RPA primers to the FLT3-691 site. Forward and reverse primers are colored in yellow and purple, relatively. (B) Comparison of amplification efficiency between different RPA primer pairs. The naked-eye results and fluorescence intensity results are shown above and below, respectively. The relative fluorescence intensity is normalized by setting the highest value to 1. A green triangle points out the most efficient F2R1 pair. (C) The identification of the amplification capacity of F2R1-mediated RPA using 1e7 1e1 gradient copies of L691 plasmid templates. This assay was conducted by 15 min of RPA and 15 min of L691-crRNA induced Cas 12 a reaction, both under $37^{\circ} \mathrm{C}$. The fluorescence intensity results and the naked-eye results are shown above and below, respectively. (D) Time-course analysis of (C)

pairwise collocation between forward and reverse primers, and they were tested to screen out the most efficient pair. In this test, 1e 2 copies of L691 plasmid templates were used to simulate the genomic DNA, and the RPA products were detected by L691-crRNA induced Cas12a reaction. After $15 \mathrm{~min}$ of RPA with different primer pairs and $15 \mathrm{~min}$ of Cas12a reaction, the strongest fluorescence signal was observed in the F2R1 pair, indicating that F2R1 is most efficient to amplify the FLT3-691 region (Figure 3B). In order to analyze its amplification capacity more specifically, we used F2R1-mediated RPA to amplify 1e7 1e1 gradient copies of L691 plasmid templates. After L691crRNA induced Cas12a reaction, the results showed that as low as 1e1 copies of L691 plasmids could be successfully detected (Figures 3C,D and Supplementary Figure S2). Together, using 15 min of F2R1mediated RPA and 15 min of L691-crRNA induced Cas12a reaction, the FLT3-F691L detection system can achieve detection of nearly single-digit copies of pure L691 DNA templates.

\section{Identification of the Sensitivity of FLT3-F691L Detection System}

Detection of low-abundance FLT3-F691L mutation may help early clinical diagnosis and avoid ineffective treatment. In order to define the sensitivity of the FLT3-F691L detection system, 1e 5 copies of plasmids with gradient F691L mutation rates were used as tested samples. The mutation rates included 100\% (pure L691), 10, 1, 0.1, 0.01\%, and 0 (WT, pure F691), generated by different proportions of L691 and F691 plasmids. These samples were amplified by RPA and then detected by L691induced Cas 12 a reaction as mentioned above. The results showed that the detection system could achieve a sensitivity of $0.1 \%$, which means detecting 100 copies of L691 templates among 99,900 copies of F691 templates. Besides, there is no significant difference between WT and NC samples, which further confirmed the high specificity of this detection system (Figures 4A,B and Supplementary Figure S3). The RPA products of these samples were also detected by F691-crRNA to show the amplification of WT templates (Figure 4A). To analyze the fluorescence change more clearly, we use a fluorescence reader to record the time course of the L691crRNA induced Cas12a reaction. The gradually increased fluorescence intensity in $0.1 \%$ sample was observed, compared with unchanged fluorescence intensity in $0.01 \%$, WT, and NC samples. Together, these results indicated that our FLT3-F691L detection system could achieve a sensitivity of $0.1 \%$ under $1 \mathrm{e} 5$ copies of templates, which is a 100 -fold improvement compared with FGS. 

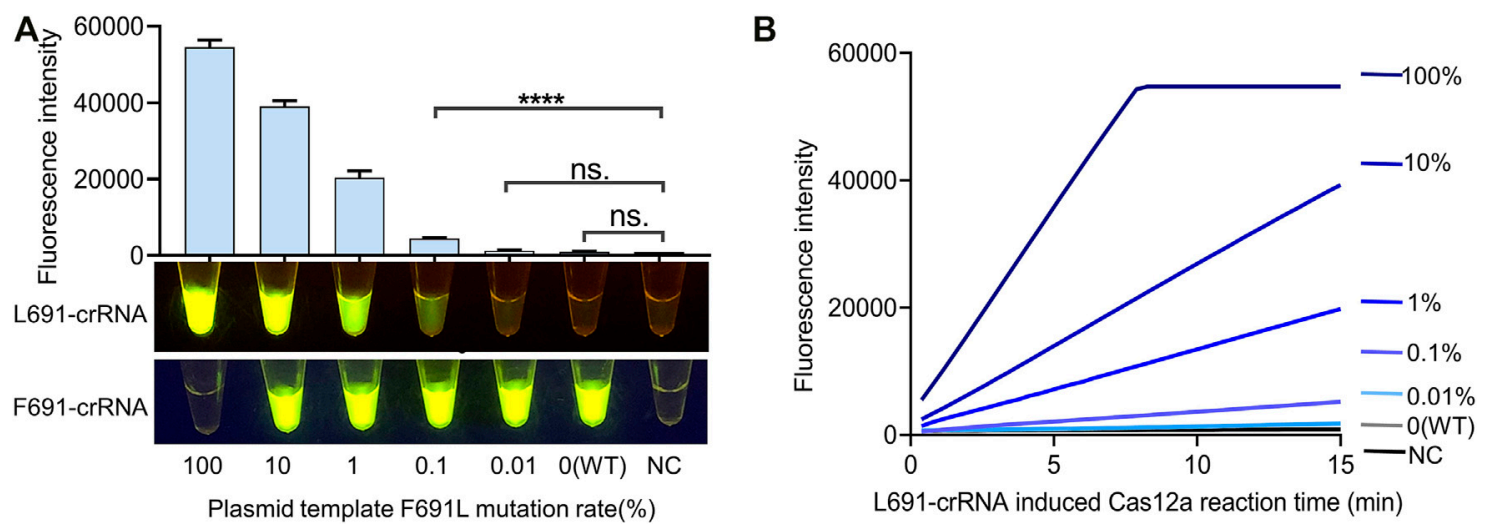

L691-crRNA induced Cas12a reaction time ( $\mathrm{min})$

FIGURE 4 | Identification of the sensitivity of FLT3-F691L detection system. (A) Sensitivity assay of the CRISPR detection system for FLT3-F691L mutation. Plasmids with gradient F691L mutation rates are used as tested samples. The detection was conducted by 15 min of F2R1-mediated RPA and 15 min of L691-crRNA induced Cas12a reaction, both under $37^{\circ} \mathrm{C}$. Final fluorescence intensity results and naked-eye results are shown above and below, respectively. The naked-eye results of F691-crRNA induced Cas12a reaction are also shown below. (B) Time-course analysis of the L691-crRNA induced Cas12a reaction in (A).
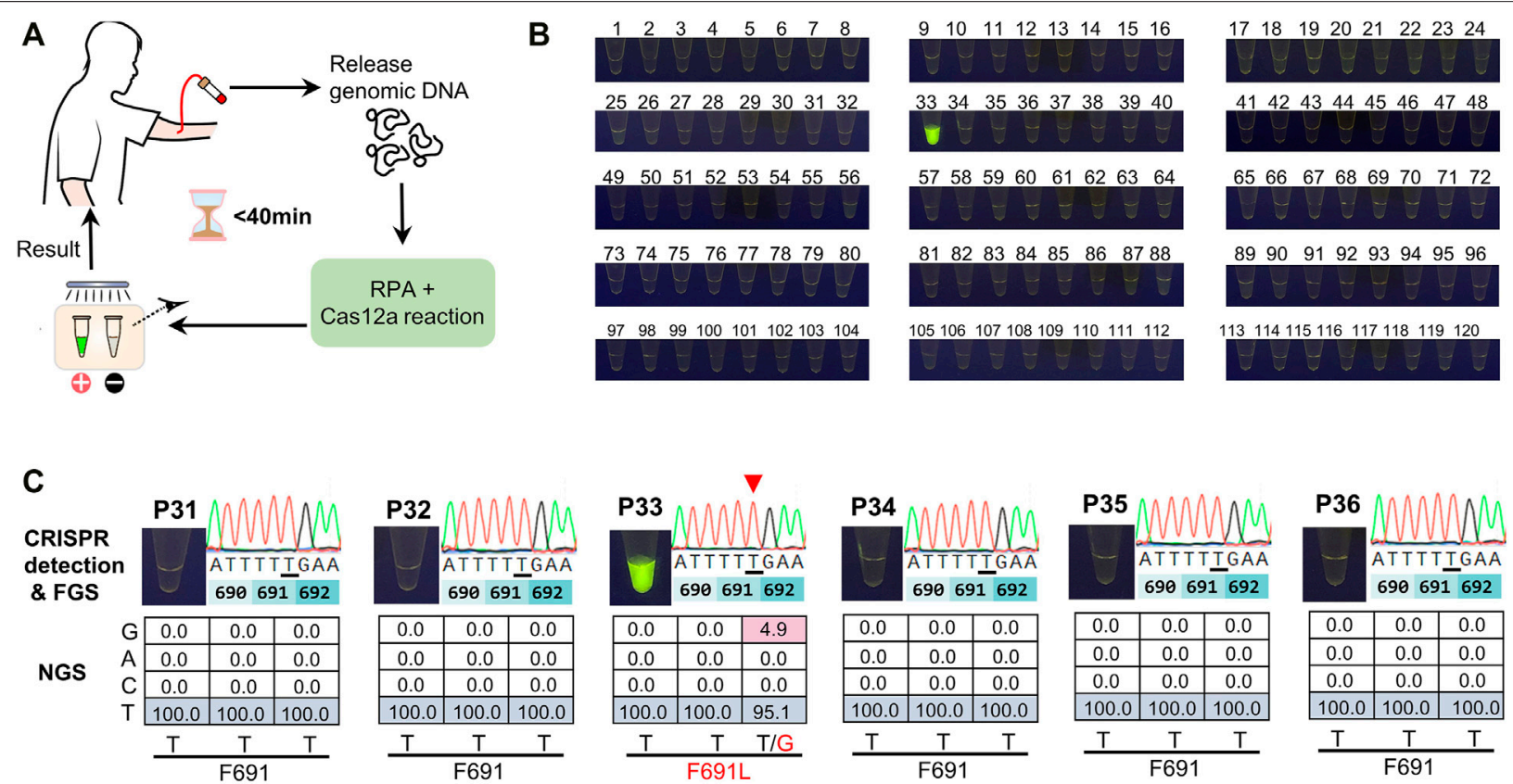

D

\begin{tabular}{lcccccc}
\hline Methods & NGS pos.(n=1) & NGS neg.(n=119) & Total & Predictive Value & Sensitivity & Specificity \\
\hline CRISPR detection pos. & 1 & 0 & 1 & $1 / 1(100)$ & $1 / 1(100)$ & --- \\
CRISPR detection neg. & 0 & 119 & 119 & $119 / 119(100)$ & --- & $119 / 119(100)$ \\
FGS pos. & 0 & 0 & 0 & $0 / 1(0)$ & $0 / 1(0)$ & --- \\
FGS neg. & 1 & 119 & 120 & $119 / 120(99.2)$ & --- & $119 / 119(100)$ \\
\hline
\end{tabular}

FIGURE 5 | Screening of AML samples for FLT3-F691L mutation by CRISPR detection. (A) Schematic diagram of the whole mutation detection process. (B) Detection results of 120 AML patients using CRISPR detection for FLT3-F691L mutation. P33 is a FLT3-F691L positive sample. (C) The CRISPR detection, FGS, and NGS results of P33 and other five FLT3-F691L negative patients. In FGS results, mutation base is underlined and pointed out by a red triangle. In NGS results, WT and mutation bases are colored in blue a red, respectively. (D) Statistical table of the sensitivity and specificity of CRISPR detection compared with FGS, using NGS as a standard reference. 


\section{Screening of AML Samples for FLT3-F691L by CRISPR Detection}

We then applied CRISPR detection to screen AML patients for FLT3-F691L positivity. Briefly, blood samples of 120 AML patients were drawn and the genomic DNA was released, then the DNA templates were used to RPA and L691-induced Cas $12 \mathrm{a}$ reaction, and finally, the results were observed by naked eyes under a blue lamp. The whole detection was completed within $40 \mathrm{~min}$ (Figure 5A). Out of the 120 samples, represented by P1 120, we found only one FLT3F691L positive case P33 (Figure 5B and Supplementary Figure S4-S6). This is consistent with the low frequency of FLT3-F691L mutation in AML populations. The NGS data of the 120 patients confirmed the CRISPR detection results, wherein P33 had a F691L mutation rate of $4.9 \%$. However, this sample was not screened out using FGS, further indicating the low sensitivity of FGS (Figure 5C). Using NGS as the gold standard, we compared CRISPR detection with FGS to detect the 120 AML patients. The statistics showed that although CRISPR detection and FGS both performed a 100\% specificity in this test, CRISPR detection displayed a much higher sensitivity than FGS (100 vs. 0\%) (Figure 5D). Together, these results verified the high efficiency, specificity, and sensitivity of the CRISPR detection method for FLT3-F691L.

\section{DISCUSSION}

In the past 2 decades since FLT3-ITD and TKD mutations were identified in acute myeloid leukemia (Nakao et al., 1996; Yamamoto et al., 2001), researchers have continued unremitting exploration on FLT3-targeted therapies. Happily, three FLT3 inhibitors including midostaurin, gilteritinib and quizartinib, have been finally approved for clinical use in 2017-2019 (Levis, 2017). It means that more than a quarter of AML patients could have a chance to improve their survival. However, the development of drug resistance has challenged the application of FLT3 inhibitors, with drug-resistant point mutations as the most important cause. Although rarely observed in AML patients, FLT3 gene F691L mutation shows universal resistance to all available FLT3 inhibitors. Therefore, F691L mutation becomes a crucial detection item for treatment decisions (Albers et al., 2013; Smith et al., 2015; Yamaura et al., 2018; Wang et al., 2021). Furthermore, earlier identification of drug-resistant mutations will help better understand the progression of the disease, and enable properly targeted treatment and more durable remissions for patients.

Although FGS and NGS have been used in clinical molecular diagnosis, they both have some drawbacks. FGS is relatively rapid but with low sensitivity, while NGS is sensitive but time-consuming and not economical. In this study, we developed an efficient, accurate, and sensitive method for FLT3-F691L detection. This method is based on RPA and Cas12a fluorescence assay; both are operated under $37^{\circ} \mathrm{C}$, without the need of large equipment and expensive reagent. Using this method, we achieved a sensitivity of $0.1 \%$ in the detection of $1 \mathrm{e} 5$ copies of plasmid templates.

Moreover, we used this CRISPR detection to test 120 AML patient samples for clinical use. For the low incidence of FLT3F691L mutation, only one F691L-mutated patient sample was screened out, which is missed by FGS detection. This positive sample and other 119 negative samples were further confirmed by NGS methods. These results indicate the advantages of our CRISPR detection method compared with FGS. Moreover, the CRISPR detection method can complete the clinical diagnosis of FLT3-F691L within $40 \mathrm{~min}$, which is much more efficient than FGS and NGS. However, due to its high sensitivity, CRISPR detection should be carefully operated in a spotless environment to avoid DNA contamination or cross-contamination among samples. In future work, to reduce the cross contamination and simplify the operation, there are some ways to try, including adding the Cas12a reaction system to the lid of the RPA tube before sealing and one-pot RPA-Cas12a detection approaches (Chen Y. et al., 2020; Feng et al., 2021; Sun et al., 2021).

In conclusion, our highly efficient and sensitive CRISPR detection provides a promising way for the clinical diagnosis of drug-resistant FLT3-F691L mutation. This method will play an important role in early screening, point-of-care and therapy options in AML treatment.

\section{DATA AVAILABILITY STATEMENT}

The raw data supporting the conclusion of this article will be made available by the authors, without undue reservation.

\section{ETHICS STATEMENT}

This study involving human blood samples and their DNA information was under the guidelines of the Declaration of Helsinki and has been reviewed and approved by the Medical Ethics Committee, Zhongnan Hospital of Wuhan University (Scientific Ethic Approval No. 2017064). The patients provided their written informed consent to participate in this study. Personal information is hidden in this study.

\section{AUTHOR CONTRIBUTIONS}

Conceptualization, YL, XW, and FZ; Methodology, YL and XW; Investigation and validation, $\mathrm{YL}$ and $\mathrm{YC}$; Resources, $\mathrm{FZ}, \mathrm{XW}, \mathrm{XH}$, and XM; Data analysis, YL and SH; Original draft preparation, YL; Article revision, FZ, XW, and XH; Supervision, FZ; Funding acquisition, $\mathrm{FZ}, \mathrm{XW}$, and $\mathrm{XM}$.

\section{FUNDING}

This study was supported by the National Natural Science Foundation of China (Grant No. 81770179, 81773249, and 82002144), and Public welfare scientific research project of Zhongshan City (2019B1015).

\section{SUPPLEMENTARY MATERIAL}

The Supplementary Material for this article can be found online at: https://www.frontiersin.org/articles/10.3389/fmolb.2021.753276/ full\#supplementary-material 


\section{REFERENCES}

Albers, C., Leischner, H., Verbeek, M., Yu, C., Illert, A. L., Peschel, C., et al. (2013). The Secondary FLT3-ITD F691L Mutation Induces Resistance to AC220 in FLT3-Itd+ AML but Retains In Vitro Sensitivity to PKC412 and Sunitinib. Leukemia 27, 1416-1418. doi:10.1038/leu.2013.14

Chen, J. S., Ma, E., Harrington, L. B., Da Costa, M., Tian, X., Palefsky, J. M., et al. (2018). CRISPR-Cas12a Target Binding Unleashes Indiscriminate SingleStranded DNase Activity. Science 360, 436-439. doi:10.1126/science.aar6245

Chen, Q., Tian, T., Xiong, E., Wang, P., and Zhou, X. (2020a). CRISPR/Cas13a Signal Amplification Linked Immunosorbent Assay for Femtomolar Protein Detection. Anal. Chem. 92, 573-577. doi:10.1021/acs.analchem.9b04403

Chen, Y., Mei, Y., Zhao, X., and Jiang, X. (2020b). Reagents-Loaded, Automated Assay that Integrates Recombinase-Aided Amplification and Cas12a Nucleic Acid Detection for a Point-of-Care Test. Anal. Chem. 92, 14846-14852. doi:10.1021/acs.analchem.0c03883

Creutzburg, S. C. A., Wu, W. Y., Mohanraju, P., Swartjes, T., Alkan, F., Gorodkin, J., et al. (2020). Good Guide, Bad Guide: Spacer Sequence-dependent Cleavage Efficiency of Cas12a. Nucleic Acids Res. 48, 3228-3243. doi:10.1093/nar/ gkz1240

Curti, L. A., Pereyra-Bonnet, F., Repizo, G. D., Fay, J. V., Salvatierra, K., Blariza, M. J., et al. (2020). CRISPR-based Platform for Carbapenemases and Emerging Viruses Detection Using Cas12a (Cpf1) Effector Nuclease. Emerging Microbes \& Infections 9, 1140-1148. doi:10.1080/22221751.2020.1763857

Daver, N., Schlenk, R. F., Russell, N. H., and Levis, M. J. (2019). Targeting FLT3 Mutations in AML: Review of Current Knowledge and Evidence. Leukemia 33, 299-312. doi:10.1038/s41375-018-0357-9

Ding, X., Yin, K., Li, Z., Lalla, R. V., Ballesteros, E., Sfeir, M. M., et al. (2020). Ultrasensitive and Visual Detection of SARS-CoV-2 Using All-In-One Dual CRISPR-Cas12a Assay. Nat. Commun. 11, 4711. doi:10.1038/s41467-02018575-6

Döhner, H., Wei, A. H., and Löwenberg, B. (2021). Towards Precision Medicine for AML. Nat. Rev. Clin. Oncol. 18, 577-590. doi:10.1038/s41571-021-00509-w

Eguchi, M., Minami, Y., Kuzume, A., and Chi, S. (2020). Mechanisms Underlying Resistance to FLT3 Inhibitors in Acute Myeloid Leukemia. Biomedicines 8, 245. doi:10.3390/biomedicines8080245

Feng, W., Peng, H., Xu, J., Liu, Y., Pabbaraju, K., Tipples, G., et al. (2021). Integrating Reverse Transcription Recombinase Polymerase Amplification with CRISPR Technology for the One-Tube Assay of RNA. Anal. Chem. 93, 12808-12816. doi:10.1021/acs.analchem.1c03456

Gootenberg, J. S., Abudayyeh, O. O., Lee, J. W., Essletzbichler, P., Dy, A. J., Joung, J., et al. (2017). Nucleic Acid Detection with CRISPR-Cas13a/C2c2. Science 356, 438-442. doi:10.1126/science.aam9321

Kiyoi, H., Kawashima, N., and Ishikawa, Y. (2020). FLT3 Mutations in Acute Myeloid Leukemia: Therapeutic Paradigm beyond Inhibitor Development. Cancer Sci. 111, 312-322. doi:10.1111/cas.14274

Levis, M. (2017). Midostaurin Approved for FLT3-Mutated AML. Blood 129, 3403-3406. doi:10.1182/blood-2017-05-782292

Li, S.-Y., Cheng, Q.-X., Liu, J.-K., Nie, X.-Q., Zhao, G.-P., and Wang, J. (2018a). CRISPR-Cas12a Has Both Cis- and Trans-cleavage Activities on SingleStranded DNA. Cell Res 28, 491-493. doi:10.1038/s41422-018-0022-x

Li, S.-Y., Cheng, Q.-X., Wang, J.-M., Li, X.-Y., Zhang, Z.-L., Gao, S., et al. (2018b). CRISPR-Cas12a-assisted Nucleic Acid Detection. Cell Discov 4, 20. doi:10.1038/ s41421-018-0028-z

Li, Y., Li, S., Wang, J., and Liu, G. (2019). CRISPR/Cas Systems towards NextGeneration Biosensing. Trends Biotechnol. 37, 730-743. doi:10.1016/ j.tibtech.2018.12.005

Lobato, I. M., and O'Sullivan, C. K. (2018). Recombinase Polymerase Amplification: Basics, Applications and Recent Advances. Trac Trends Anal. Chem. 98, 19-35. doi:10.1016/j.trac.2017.10.015

Ma, P., Meng, Q., Sun, B., Zhao, B., Dang, L., Zhong, M., et al. (2020). MeCas12a, a Highly Sensitive and Specific System for COVID-19 Detection. Adv. Sci. 7, 2001300. doi:10.1002/advs.202001300
Myhrvold, C., Freije, C. A., Gootenberg, J. S., Abudayyeh, O. O., Metsky, H. C., Durbin, A. F., et al. (2018). Field-deployable Viral Diagnostics Using CRISPRCas13. Science 360, 444-448. doi:10.1126/science.aas8836

Nakao, M., Yokota, S., Iwai, T., Kaneko, H., Horiike, S., Kashima, K., et al. (1996). Internal Tandem Duplication of the Flt3 Gene Found in Acute Myeloid Leukemia. Leukemia 10, 1911-1918.

Smith, C. C., Zhang, C., Lin, K. C., Lasater, E. A., Zhang, Y., Massi, E., et al. (2015). Characterizing and Overriding the Structural Mechanism of the QuizartinibResistant FLT3 "Gatekeeper" F691L Mutation with PLX3397. Cancer Discov. 5, 668-679. doi:10.1158/2159-8290.CD-15-0060

Sun, Y., Yu, L., Liu, C., Ye, S., Chen, W., Li, D., et al. (2021). One-tube SARS-CoV-2 Detection Platform Based on RT-RPA and CRISPR/Cas12a. J. Transl Med. 19, 74. doi:10.1186/s12967-021-02741-5

Tarver, T. C., Hill, J. E., Rahmat, L., Perl, A. E., Bahceci, E., Mori, K., et al. (2020). Gilteritinib Is a Clinically Active FLT3 Inhibitor with Broad Activity against FLT3 Kinase Domain Mutations. Blood Adv. 4, 514-524. doi:10.1182/ bloodadvances.2019000919

Wang, P., Xiao, X., Zhang, Y., Zhang, B., Li, D., Liu, M., et al. (2021). A Dual Inhibitor Overcomes Drug-Resistant FLT3-ITD Acute Myeloid Leukemia. J. Hematol. Oncol. 14, 105. doi:10.1186/s13045-021-01098-y

Wang, X., Ji, P., Fan, H., Dang, L., Wan, W., Liu, S., et al. (2020a). CRISPR/Cas12a Technology Combined with Immunochromatographic Strips for Portable Detection of African Swine Fever Virus. Commun. Biol. 3, 62. doi:10.1038/ s42003-020-0796-5

Wang, X., Shang, X., and Huang, X. (2020b). Next-generation Pathogen Diagnosis with CRISPR/Cas-based Detection Methods. Emerging Microbes \& Infections 9, 1682-1691. doi:10.1080/22221751.2020.1793689

Wang, X., Zhong, M., Liu, Y., Ma, P., Dang, L., Meng, Q., et al. (2020c). Rapid and Sensitive Detection of COVID-19 Using CRISPR/Cas12a-based Detection with Naked Eye Readout, CRISPR/Cas12a-NER. Sci. Bull. 65, 1436-1439. doi:10.1016/j.scib.2020.04.041

Xu, H., Zhang, X., Cai, Z., Dong, X., Chen, G., Li, Z., et al. (2020). An Isothermal Method for Sensitive Detection of Mycobacterium tuberculosis Complex Using Clustered Regularly Interspaced Short Palindromic Repeats/Cas12a Cis and Trans Cleavage. J. Mol. Diagn. 22, 1020-1029. doi:10.1016/j.jmoldx.2020.04.212

Yamamoto, Y., Kiyoi, H., Nakano, Y., Suzuki, R., Kodera, Y., Miyawaki, S., et al. (2001). Activating Mutation of D835 within the Activation Loop of FLT3 in Human Hematologic Malignancies. Blood 97, 2434-2439. doi:10.1182/ blood.v97.8.2434

Yamaura, T., Nakatani, T., Uda, K., Ogura, H., Shin, W., Kurokawa, N., et al. (2018). A Novel Irreversible FLT3 Inhibitor, FF-10101, Shows Excellent Efficacy against AML Cells with FLT3 Mutations. Blood 131, 426-438. doi:10.1182/blood-2017-05-786657

Zhou, W., Hu, L., Ying, L., Zhao, Z., Chu, P. K., and Yu, X.-F. (2018). A CRISPRCas9-Triggered Strand Displacement Amplification Method for Ultrasensitive DNA Detection. Nat. Commun. 9, 5012. doi:10.1038/s41467-018-07324-5

Conflict of Interest: The authors declare that the research was conducted in the absence of any commercial or financial relationships that could be construed as a potential conflict of interest.

Publisher's Note: All claims expressed in this article are solely those of the authors and do not necessarily represent those of their affiliated organizations, or those of the publisher, the editors and the reviewers. Any product that may be evaluated in this article, or claim that may be made by its manufacturer, is not guaranteed or endorsed by the publisher.

Copyright (c) 2021 Liu, Chen, Huang, Ma, Huang, Wang and Zhou. This is an openaccess article distributed under the terms of the Creative Commons Attribution License (CC BY). The use, distribution or reproduction in other forums is permitted, provided the original author(s) and the copyright owner(s) are credited and that the original publication in this journal is cited, in accordance with accepted academic practice. No use, distribution or reproduction is permitted which does not comply with these terms. 